\title{
Uniform Dispersion of Multiwalled Carbon Nanotubes in Copper Matrix Nanocomposites Using Metal Injection Molding Technique
}

\author{
Ali Samer Muhsan, ${ }^{1}$ Faiz Ahmad, ${ }^{1}$ Norani M. Mohamed, ${ }^{2}$ \\ Puteri Sri Melor Megat Yusoff, ${ }^{1}$ and Muhammad Rafi Raza ${ }^{3}$ \\ ${ }^{1}$ Mechanical Engineering Department, Universiti Teknologi PETRONAS (UTP), Bandar Seri Iskandar, \\ 31750 Tronoh, Perak Darul Ridzuan, Malaysia \\ ${ }^{2}$ Centre of Innovative Nanostructures and Nanodevices (COINN), UTP, Bandar Seri Iskandar, 31750 Tronoh, \\ Perak Darul Ridzuan, Malaysia \\ ${ }^{3}$ Department of Mechanical \& Materials Engineering, Universiti Kebangsaan, 43600 UKM Bangi, \\ Selangor Darul Ehsan, Malaysia
}

Correspondence should be addressed to Faiz Ahmad; faizahmadster@gmail.com

Received 10 March 2013; Accepted 28 August 2013

Academic Editors: A. Lockamy and B.-K. Min

Copyright (C) 2013 Ali Samer Muhsan et al. This is an open access article distributed under the Creative Commons Attribution License, which permits unrestricted use, distribution, and reproduction in any medium, provided the original work is properly cited.

\begin{abstract}
This work presents a novel fabrication approach of multiwalled carbon nanotubes (MWNTs) reinforced copper $(\mathrm{Cu})$ matrix nanocomposites. A combination of nanoscale dispersion of functionalized MWNTs in low viscose media of dissolved paraffin wax under sonication treatment followed by metal injection molding (MIM) technique was adopted. MWNTs contents were varied from 0 to $10 \mathrm{vol} \%$. Information about the degree of purification and functionalization processes, evidences on the existence of the functional groups, effect of sonication time on the treated MWNTs, and microstructural analysis of the fabricated Cu/MWNTs nanocomposites were determined using TEM, EDX, FESEM, and Raman spectroscopy analysis. The results showed that the impurities of the pristine MWNTs such as Fe, Ni catalyst, and the amorphous carbon have been significantly removed after purification process. Meanwhile, FESEM and TEM observations showed high stability of MWNTs at elevated temperatures and uniform dispersion of MWNTs in $\mathrm{Cu}$ matrix at different volume fractions and sintering temperatures $\left(950,1000 \& 1050^{\circ} \mathrm{C}\right)$. The experimentally measured thermal conductivities of $\mathrm{Cu} / \mathrm{MWNTs}$ nanocomposites showed remarkable increase (11.25\% higher than sintered pure $\mathrm{Cu}$ ) with addition of 1 vol.\% MWNTs, and slight decrease below the value of sintered $\mathrm{Cu}$ at 5 and 10 vol.\% MWNTs.
\end{abstract}

\section{Introduction}

With continued demand for high-performance electronic devices with low cost, small size and more efficient electronic systems, thermal challenges became a serious concern in electronic packaging design [1]. More transistors means more heat is generated within these systems. High-operating temperature decreases the overall reliability or even permanently damages the entire electronic system [2]. Therefore, a new thermal management solution is required to provide costeffective means of dissipating heat from next generation microelectronic devices [3]. In response to these critical needs, high-performance heat sink nanocomposite made of copper reinforced by multiwalled carbon nanotubes is proposed.

The superior physical, mechanical, and thermal properties of MWNTs have led it to have very high impact on developing new generations of nanocomposite materials with enhanced functionality and wide range of applications [4]. However, incorporation of MWNTs in metal matrix has its own barriers and difficulties that might destroy the desired properties of MWNTs [5].

Meanwhile, dispersion of MWNTs in metal matrix nanocomposites is known as the most difficult challenge in fabricating MWNTs based metal matrix nanocomposite. 
This can be attributed to the following factors: (1) the incompatibility in phases between metal matrix and MWNTs; (2) the large difference in density; (3) bundling and tangling phenomenon of MWNTs that occurs due to the high surface area of MWNTs associated with van der Waal forces between individual MWNTs. Experimentally, most of the previous works showed decrease in the physical properties of MWNTs based nanocomposites mostly due to the MWNTs clustering phenomenon [6-9].

Therefore, several techniques have been suggested to improve the dispersion of MWNTs. Unfortunately, most of these techniques were limited to polymer nanocomposites, while only few researchers have focused on improving MWNTs dispersion in metal matrix nanocomposite. Even so, their results did not meet their expectations. For example, Chu et al. [7] studied the effect of incorporating different amounts of MWNTs in $\mathrm{Cu}$ matrix using ball milling mixing technique on thermal conductivity. They found that there was no enhancement in the thermal conductivity and they proposed that this behaviour was probably due to the random orientation of MWNTs, the existence of interfacial thermal resistance between MWNTs and copper particles associated with low phase contrast ratio of MWNTs to copper, as well as the poor dispersion of MWNTs in the copper matrix. Similar results were reported by Kim et al. [9] after reinforcing $\mathrm{Cu}$ with nickel-coated single-walled carbon nanotubes (SWNTs). They suggested that the existence of grain boundaries and defects in the polycrystals of the composite, resulting in lower thermal conductivity compared to the matrix. Cho et al. [10] used a combination of wet mixing process accelerated by hetroaggregation method using acid-treated MWNTs followed by Spark Plasma Sintering technique. Their results, however, showed no enhancement in thermal conductivity with MWNTs contents higher than 1 vol.\%. On the other hand, some researchers have carried out functionalization process on MWNTs to enhance the dispersion of the purified MWNTs and create sidewalls groups such as $\mathrm{COOH}$ that have the potential to bind MWNTs to the metal matrix [11-15]. For example, Kim et al. [11] reported the observation of enhanced mechanical properties of $\mathrm{MWNT} / \mathrm{Cu}$ nanocomposites using a molecular mixing technique. They also observed oxygen atoms at the interface between MWNTs and $\mathrm{Cu}$ matrix and proposed that these oxygen molecules had a positive effect on the interfacial strength.

Therefore, this study aims to develop a new dispersing technique of multiwalled carbon nanotubes reinforced copper matrix nanocomposites that ensures a homogeneous dispersion of MWNTs in Cu matrix. This technique is basically a multilevel mixing approach of nanoscale dispersion of the functionalized MWNTs in $\mathrm{Cu} / \mathrm{MWNT}$ feedstock followed by MIM process.

\section{Experimental Methods}

2.1. Materials. Commercial Cu powder particles with diameter ranging of 15-20 $\mu \mathrm{m}$ were purchased from Sandvik Osprey LTD, UK. Multiwalled carbon nanotubes with diameter of
60-80 $\mathrm{nm}$ and 5-15 $\mu \mathrm{m}$ in length were purchased from Shenzhen Nano-Technologies Port Co., Ltd., China. The purity of MWNTs was $95-98 \%$ with ash content of $\leq 0.2$ wt. $\%$.

2.2. Nanoscale Dispersion Process. In this method, MWNTs were first purified and functionalized using concentrated acids with the assist of heating and sonication process. MWNTs were immersed in an acid solution of $\mathrm{H}_{2} \mathrm{SO}_{4}$ (98\%) and $\mathrm{HNO}_{3}(67 \%)$ in a ratio of $3: 1$ for different sonication times $(0.5,2.75$ and $5 \mathrm{~h})$ under temperature of $70^{\circ} \mathrm{C}$. Functionalization has been applied to enhance the dispersion of purified MWNTs and create sidewalls groups that have the potential to bind MWNTs to the $\mathrm{Cu}$ matrix.

After MWNTs being purified and functionalized in concentrated acids under sonication treatment, MWNTs were washed by distilled water and dried at $120^{\circ} \mathrm{C}$ for $24 \mathrm{~h}$. MWNTs were mixed again with low viscous liquid media of dissolved paraffin wax (PW) diluted in heptane solvent along with magnetic stirring. This was followed by sonication agitation process to ensure uniform dispersion of MWNTs in PW solution. The dried MWNTs/PW mixture was then mixed with the other components of the binder system (Polyethylene, PE; Stearic Acid, SA) in Z-blade mixer at $160^{\circ} \mathrm{C}$. This step provided a high-viscous liquid media with high shear forces that allowed MWNTs to be mixed properly by dispersing the MWNTs clusters with uniform dispersion into the molten binder system. The copper powder was added gradually during the mixing process to form $\mathrm{Cu}-\mathrm{MWNT}$ Binder feedstock as illustrated in Figure 1.

2.3. Metal Injection Molding Process. Metal injection molding (MIM) technique involves four main stages: feedstock preparation; injection molding of the prepared feedstock; binder removal or debinding process (solvent and thermal debinding) to get a shaped porous metallic part; sintering process in which the debinded samples are condensed to form pure or composite metallic products [6]. In this technique, the prepared feedstock in the previous stage was granulated to small pieces to be plasticized in a heated barrel of injection molding machine, and, then, a controlled volume of the molten feedstock is injected from the plasticator under pressure into a closed mould, with solidification beginning on the mould's cavity wall to get the desired shape. The injection moulded samples are called green samples. To completely remove the binder from the green samples, two consecutive processes of solvent and thermal debinding were applied. After the binder is being totally removed, sintering process under Argon atmosphere with different sintering temperature of 950,1000 and $1050^{\circ} \mathrm{C}$ was carried out as shown in Figure 2.

\section{Results and Discussion}

3.1. TEM and EDX Analysis. Figure 3 shows the transmission electron micrograph (TEM) of MWNTs before (a) and after (b) purification process. It can be seen that the impurities of the pristine MWNTs, such as Fe, Ni catalyst, and the amorphous carbon have been successfully removed after the 

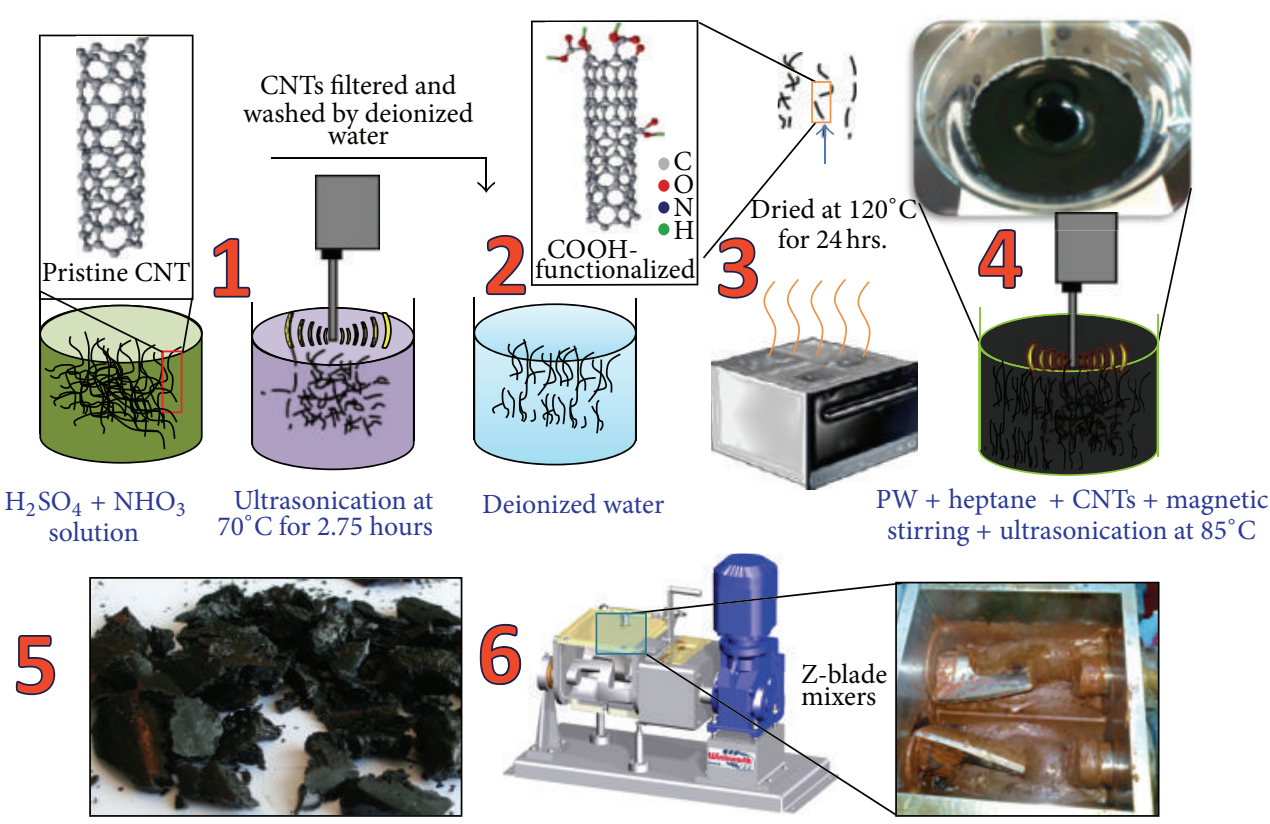

Dried $(\mathrm{PW}+\mathrm{CNTs})$

$(\mathrm{PW}+\mathrm{PE}+\mathrm{SA}+\mathrm{CNTs}+\mathrm{Cu})$

Feedstock preparation

FIgURE 1: Nanoscale dispersion process of MWNTs in Paraffin Wax (PW).

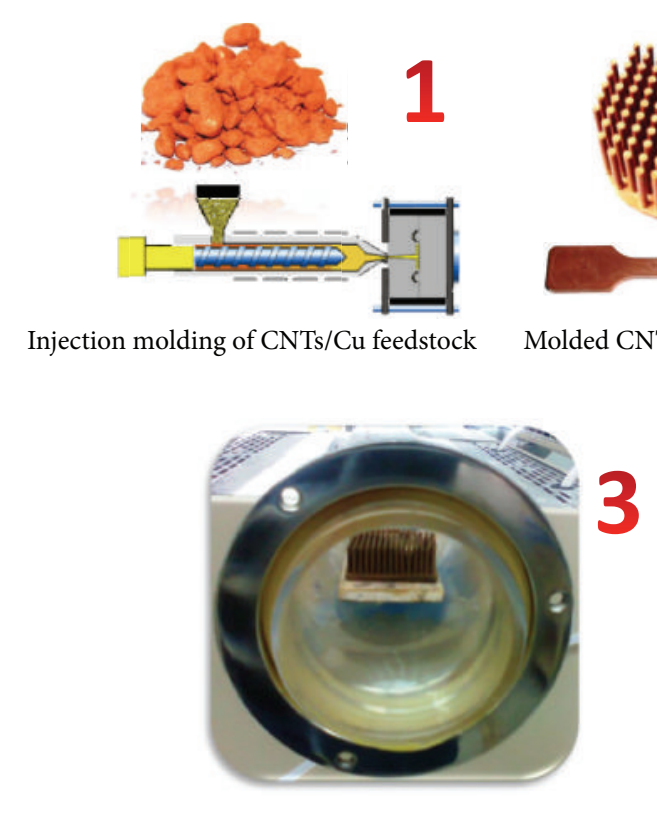

Thermal debinding and sintering process

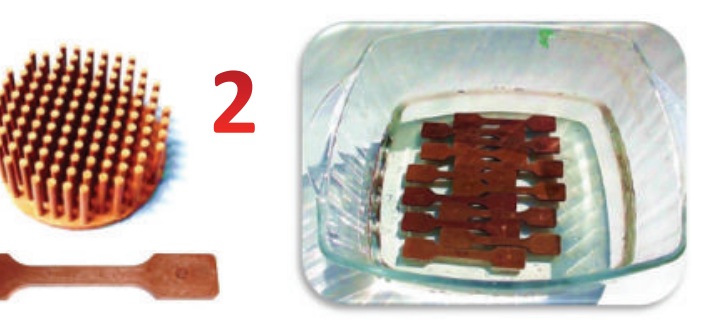

Solvent debinding process (heptane)

FIgURE 2: Metal injection process of MWNTs/Cu nanocomposites.

purification and sonication process. These results were further proved by Energy dispersive X-ray spectroscopy (EDX) and Raman microscope inspection. EDX results (Table 1) show that Fe catalyst was completely eliminated and $\mathrm{Ni}$ was reduced significantly after acid treatment. However, longer time of sonication treatment may result in aggressive oxidation of the MWNT surface that can degrade the MWNTs. Therefore, in order to test the effect of sonication process on the MWNTs structure, different sonication hours of $0.5,2.75$, and 5 were applied. The results revealed that the sonication time of $2.75 \mathrm{~h}$ was recorded as the optimal parameter.

\subsection{Raman Spectroscopy and SEM Characterization Tests} of Functionalized MWNTs. Raman spectroscopy revealed necessary information on the evolution of MWNTs after chemical or physical treatment [16]. Figure 4 illustrates the 


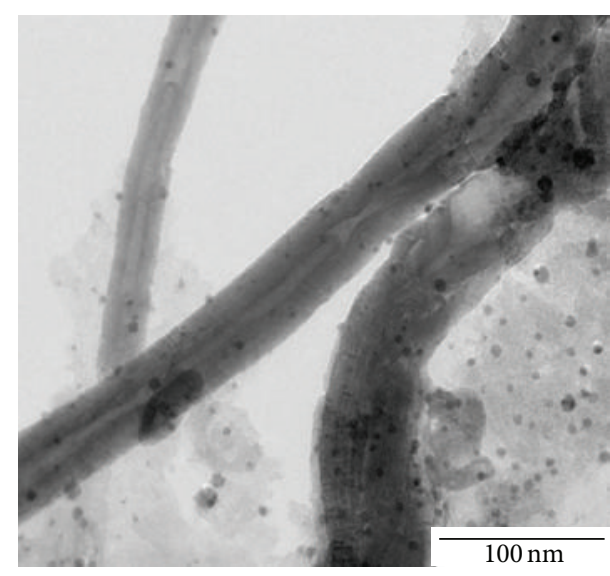

(a)

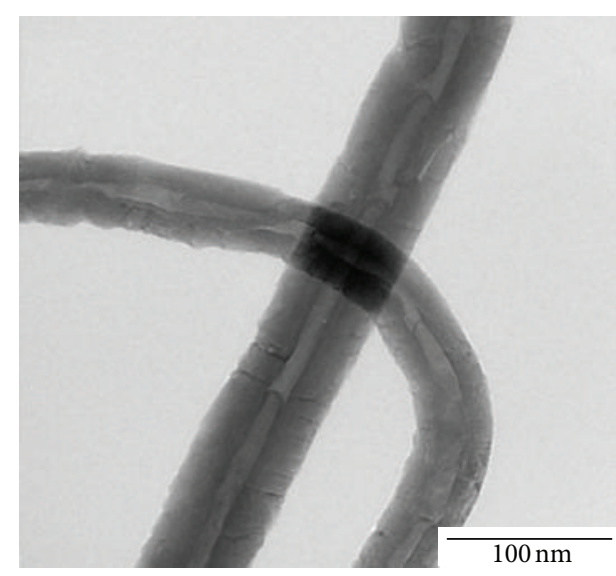

(b)

Figure 3: TEM images of (a) Pristine MWNTs and (b) Purified MWNTs with sonication treatment for $2.75 \mathrm{~h}$.

Raman spectra of pristine MWNTs (Raw MWNTs), acid treated MWNTs with sonication time of $0.5,2.75$ and $5 \mathrm{~h}$. The $\mathrm{D} / \mathrm{G}$ ratio represents the defect density in graphitic structures. As clearly shown in Figure 4, there were two peaks at $\sim 1330 \mathrm{~cm}^{-1}$ (D band) and the other at $\sim 1570 \mathrm{~cm}^{-1}$ (G band). By the comparison of the $I_{\mathrm{D}} / I_{\mathrm{G}}$ ratios of these spectra, it could be seen that this ratio was varied as sonication time increased. The pristine MWNTs had the lowest $I_{\mathrm{D}} / I_{\mathrm{G}}$ ratios of 0.838 . The $I_{\mathrm{D}} / I_{\mathrm{G}}$ ratios gradually increased to 0.848 , 0.855 , and 0.861 of the MWNTs treated under sonication process of $0.5,2.75$, and 5 hours, respectively. The amount of defects apparently increased in MWNTs after functionalization process, especially the one after 5 hours of sonication treatment. These results were further confirmed by FESEM characterization, as shown in Figure 5. This Figure includes four FESEM images which corresponding to (a) the pristine MWNTs, (b) acid treated MWNTs (functionalized MWNTs) with sonication process of 0.5 , (c) 2.75, and (d) 5 hours. Although EDX inspection test showed higher impurities removal at $5 \mathrm{~h}$ sonication treatment (Table 1), Figure 5(d) revealed that clear damages were occurred on the MWNTs structure after 5 hours of sonication treatment. Therefore, based on these results, the sonication treatment time chosen was 2.75 hours.

\subsection{Microstructural Analysis of Cu/MWNTs Nanocomposites.} Figure 6 shows the FESEM images of Cu/MWNTs nanocomposites demonstrating the quality of MWNTs dispersion in Cu matrix at different fabrication stages. Figures 6(a) and 6(b) illustrate the FESEM of Cu/MWNTs green (before debinding process) and sintered parts, respectively. These parts were fabricated without utilizing the newly developed technique, and they are presented here for comparison purposes. It clearly showed that MWNTs were agglomerated between the $\mathrm{Cu}$ particles and formed porosities rather than enhancing the interfacial bonding. Figures 6(c) and 6(d) represent the green and brown (thermal debinded) $\mathrm{Cu} / \mathrm{MWNT}$ parts after applying the new dispersion technique. Figures 6(e), 6(f), and $6(\mathrm{~g})$ show the fractured surfaces of the sintered $\mathrm{Cu} / \mathrm{MWNT}$
TABLE 1: EDX results of acid treated MWNTs under different Sonication Hours.

\begin{tabular}{lcc}
\hline Element & Weight $\%$ & Atomic\% \\
\hline & 0.5 h Sonication treatment & \\
$\mathrm{C}$ & 94.22 & 96.52 \\
$\mathrm{O}$ & 4.04 & 3.11 \\
$\mathrm{Fe}$ & 0.45 & 0.10 \\
$\mathrm{Ni}$ & 1.29 & 0.27 \\
\hline & & \\
$\mathrm{C}$ & 90.56 & 93.40 \\
$\mathrm{O}$ & 7.95 & 6.16 \\
$\mathrm{Fe}$ & - & - \\
$\mathrm{S}$ & 0.73 & 0.28 \\
$\mathrm{Ni}$ & 0.76 & 0.16 \\
\hline & & \\
$\mathrm{C}$ & 88.93 & 91.82 \\
$\mathrm{O}$ & 10.25 & 7.95 \\
$\mathrm{Fe}$ & - & - \\
$\mathrm{S}$ & 0.32 & 0.12 \\
$\mathrm{Ni}$ & 0.50 & 0.10 \\
\hline
\end{tabular}

parts at different sintering temperature (950, 1000, and $1050^{\circ} \mathrm{C}$, resp.). Figure $6(\mathrm{~h})$ presents a TEM nanograph, which in turn illustrates the incorporation of the individual MWNT into the $\mathrm{Cu}$ particles. It revealed that the mechanical damages in MWNTs structure were very few. It also showed a good interfacial bonding at $\mathrm{Cu} / \mathrm{MWNT}$ interface.

In general, we can clearly note that MWNTs dispersion has been significantly improved after utilizing the new technique of nanoscale dispersion. This can be attributed first to the functionalization process that provides $\mathrm{COOH}$ groups on the outer surfaces of the MWNTs. In addition, sonication process generates energy in different forms on the MWNTs clusters, these energies are oscillating zones of high temperature with extremely rapid heating and cooling rates; alternating impulses of very high pressure; formation of 


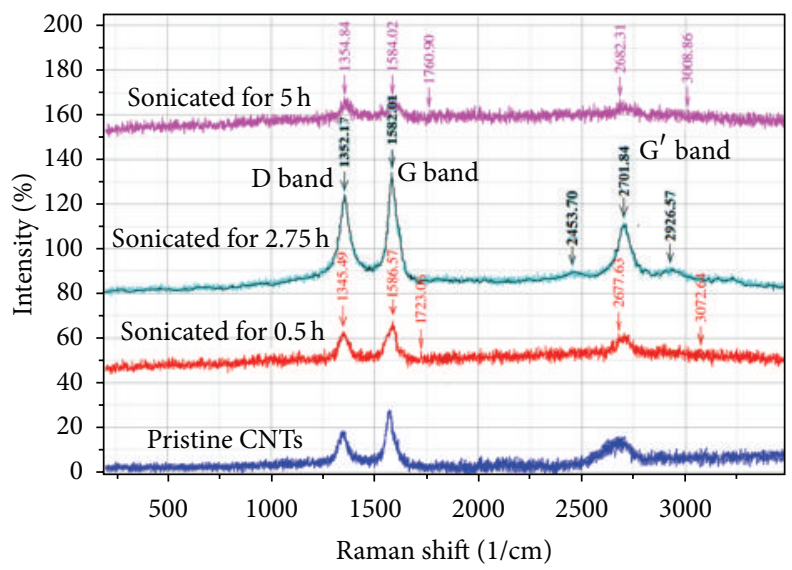

FIGURE 4: Raman spectra of raw MWNTs and functionalized MWNTs using a mixture of $\mathrm{H}_{2} \mathrm{SO}_{4}: \mathrm{NHO}_{3}(3: 1)$ under different sonication's dwell time.

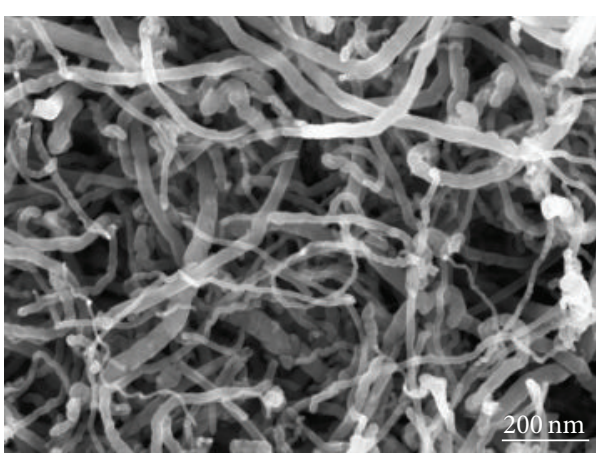

(a)

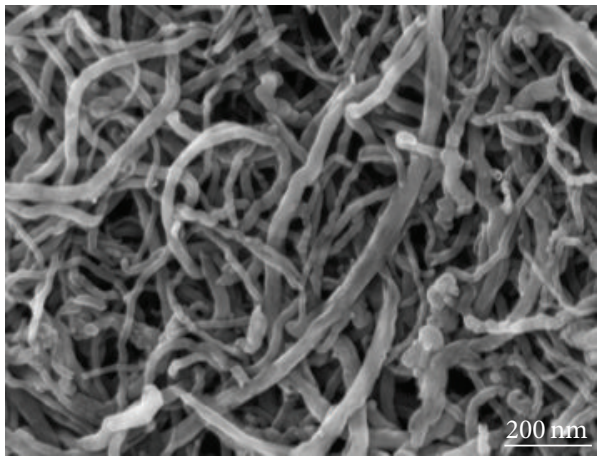

(c)

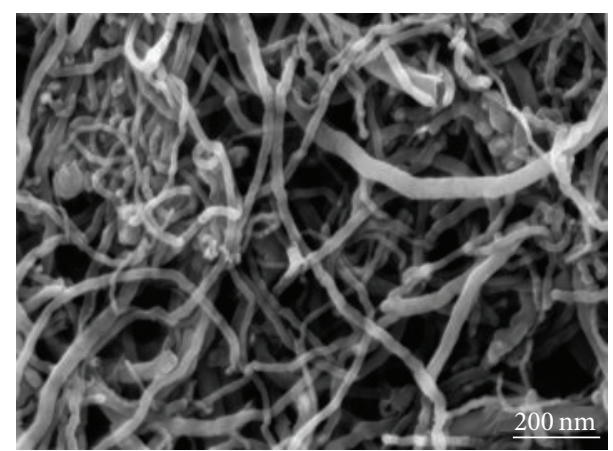

(b)

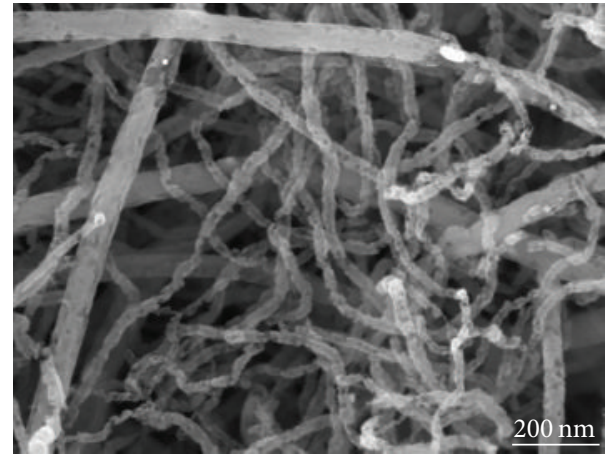

(d)

FIGURE 5: FESEM images of raw and functionalized MWNTs. (a) pristine MWNTs, (b) sonication treatment for 0.5 hour, (c) treated for 2.75 hours and (d) treated for 5 hours.

microbubbles throughout the liquid medium of MWNTs that growth and collapse on the MWNTs surfaces. These energies are sufficient to push the individual MWNT apart and overcome the van der Waal forces between MWNTs, which is considered as the main reason of the MWNTs clustering phenomenon [16-18]. Meanwhile, MIM technique provided high-shear forces from the binder system components during feedstock preparation that disperse and exfoliate the MWNTs clusters into the $\mathrm{Cu}$ matrix homogeneously. This indicates that nanoscale dispersion method combined with MIM technique is a very effective method for achieving uniform distribution of MWNTs in metal matrix nanocomposites.

3.4. Relative Density. Figure 7 shows the effect of sintering temperature on the relative density of MWNTs/Cu nanocomposites at different dwell time and MWNTs vol. $\%$. At $1050^{\circ} \mathrm{C}$ sintering temperature and dwell time of $60 \mathrm{~min}$, the measured relative densities were about $94.8 \%, 93.5 \%, 90.6 \%$, and $89 \%$ for pure copper, 1,5 , and 10 vol.\% MWNTs, respectively. In general, the relative densities of MWNTs/Cu nanocomposites 


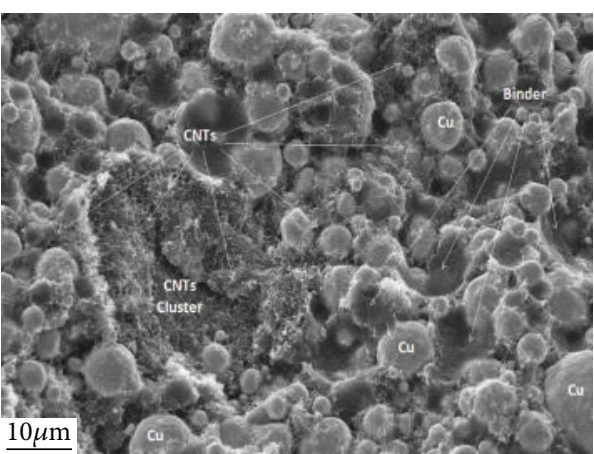

(a)

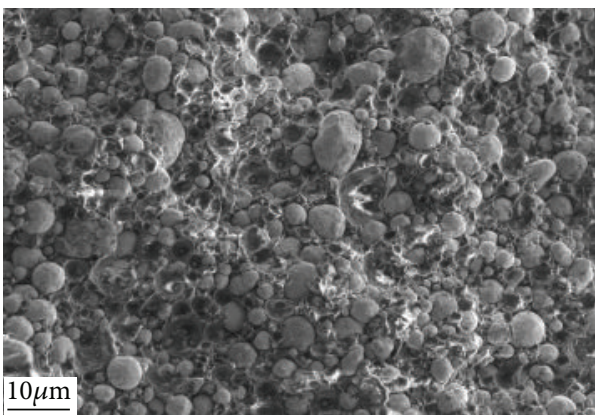

(c)

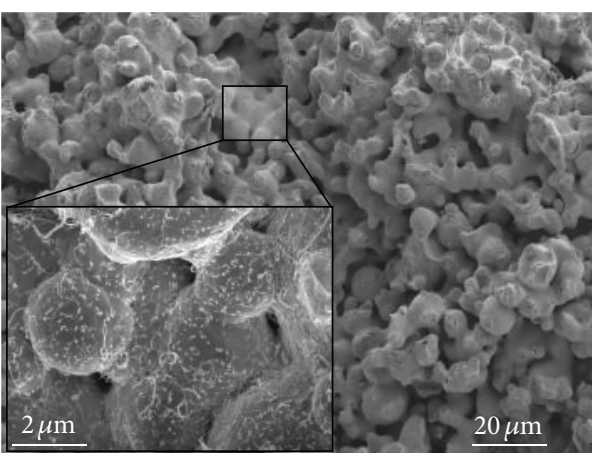

(e)

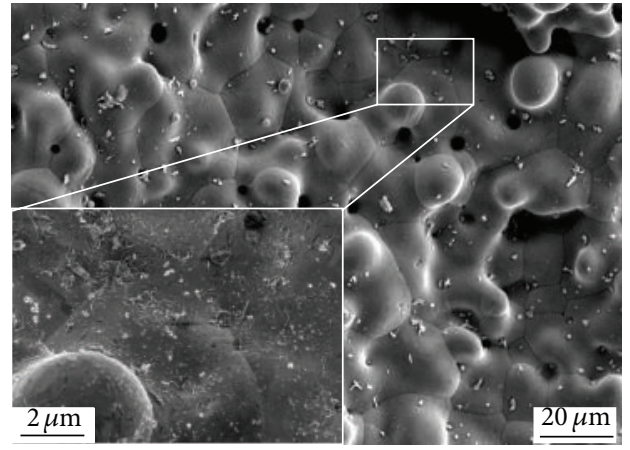

(g)

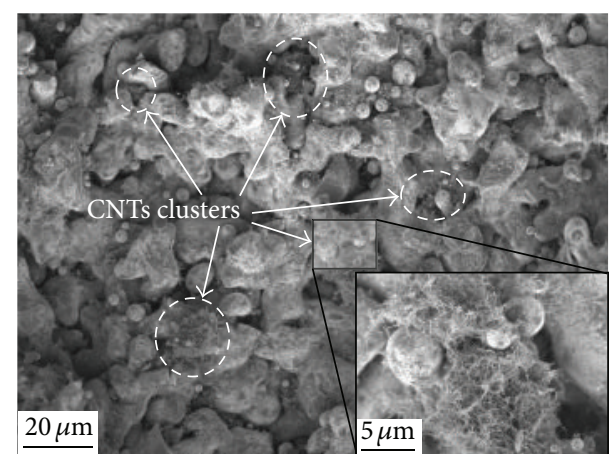

(b)

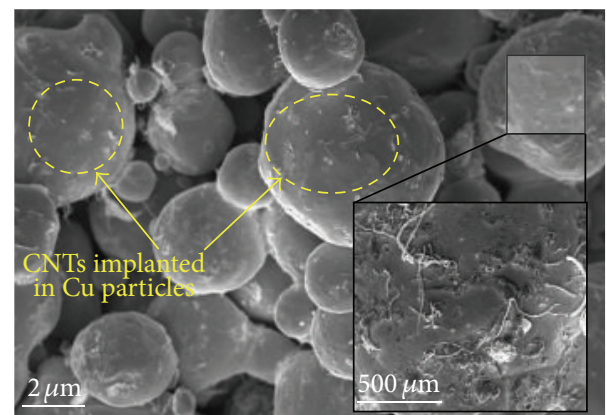

(d)

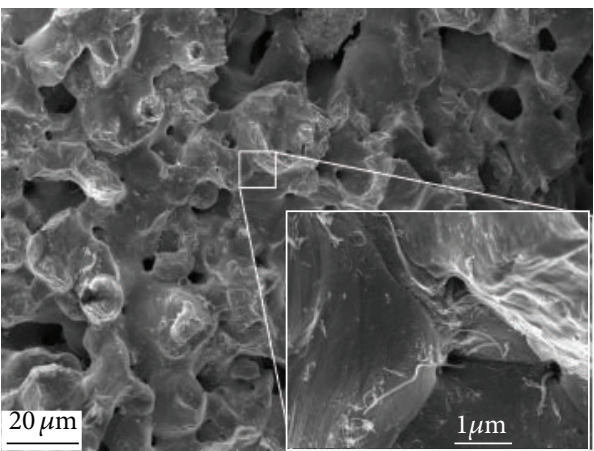

(f)

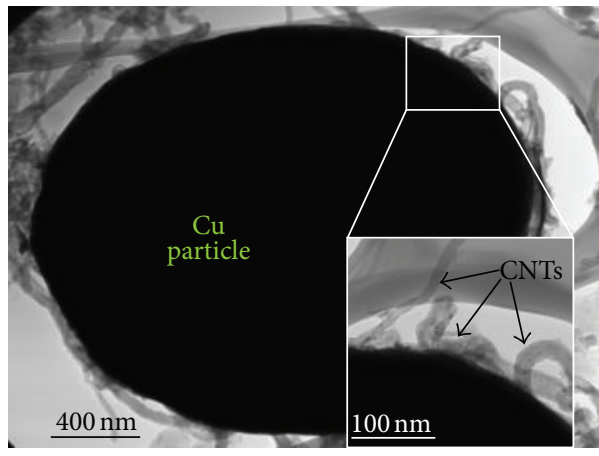

(h)

FIGURE 6: FESEM micrograph of sintered Cu-10 vol.\% MWNTs nanocomposites. (a) and (b) are the FESEM of Cu/MWNTs green and sintered parts, respectively, before applying the new dispersion technique, (c) and (d) represent the green and brown samples of Cu/MWNTs sintered at 950 and $1000^{\circ} \mathrm{C}$, respectively, both are after applying the new dispersion technique. Images (e), (f) and (g) show the fractured surfaces of the sintered $\mathrm{Cu} / \mathrm{MWNTs}$ parts at 950,1000 and $1050^{\circ} \mathrm{C}$, respectively. (h) TEM nano-graph showing good bonding at the interface between functionalized MWNTs and $\mathrm{Cu}$ particles. 


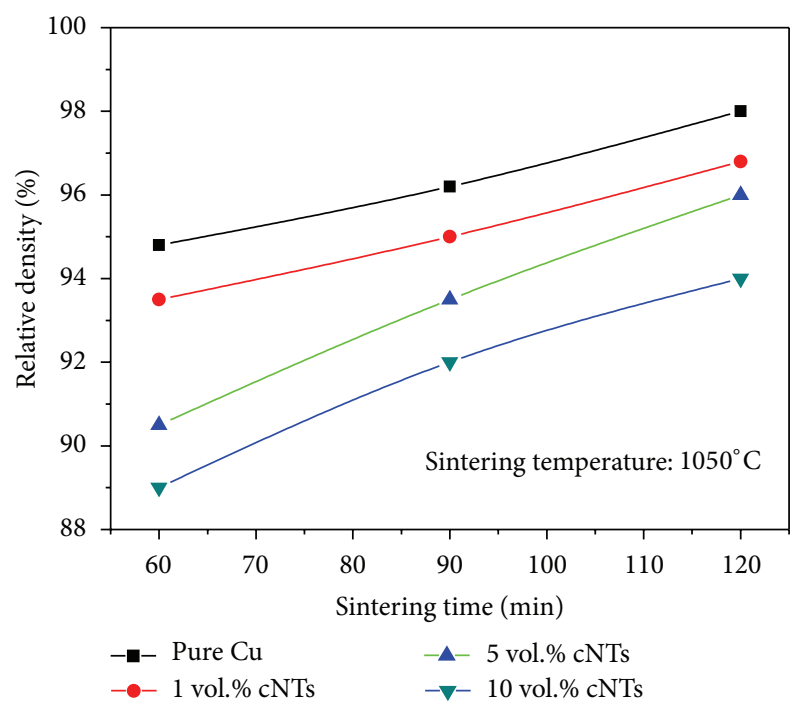

FIgURE 7: Relative densities of MWNTs/Cu nanocomposites.

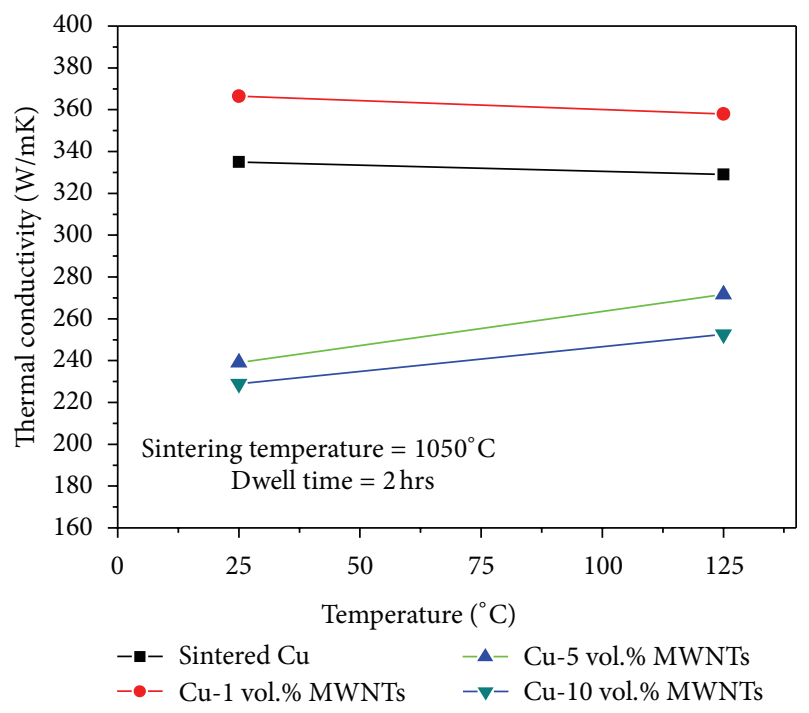

FIgURE 8: Thermal conductivities of MWNTs/Cu composites as a function of MWNTs volume fraction with two different measuring temperatures $\left(25\right.$ and $\left.125^{\circ} \mathrm{C}\right)$.

were lower than the pure $\mathrm{Cu}$ matrix due to accumulation of MWNTs at the grain boundaries of the $\mathrm{Cu}$ matrix, which is the basis of the pores creation. These accumulations increased by increase the MWNTs volume fraction. Similar behaviour was observed at $90 \mathrm{~min}$ and $120 \mathrm{~min}$ dwell times. However, it can also be noted that there is an increasing in all relative densities results as the dwell time increased regardless at which sintering temperature were they fabricated.

3.5. Thermal Conductivity Measurement. Thermal conductivities of MWCNTs/Cu composites were measured using NETZSCH model LFA 447 NanoFlash apparatus (LFA447, Netzsch, Germany). Figure 8 demonstrates the thermal conductivities of MWNTs/Cu nanocomposites as a function of MWNTs volume fraction with two different measuring temperatures $\left(25\right.$ and $\left.125^{\circ} \mathrm{C}\right)$. The $\mathrm{MWNTs} / \mathrm{Cu}$ thermal conductivities were measured in direction perpendicular to the injection molding axis and it showed varied values as MWNTs content increased. The highest value was recorded at $\mathrm{Cu}-1$ vol.\% MWNTs $(366.516 \mathrm{~W} / \mathrm{mK})$ sintered at $1050^{\circ} \mathrm{C}$ for 120 minutes. This value corresponds to an increase of $\sim 32 \mathrm{~W} / \mathrm{mK}(11.25 \%)$ over that of the sintered copper $(\mathrm{Cu}$ matrix). It is worth mentioning that the sintered copper powder with polycystic structure has lower thermal conductivity $(330 \mathrm{~W} / \mathrm{mK})$ than the monocrystal copper $(401 \mathrm{~W} / \mathrm{mK})$, and this most probably is due to the existence of grain boundary and defects in polycrystals [19]. It can also be seen that thermal conductivities of sintered copper and $\mathrm{Cu}-1$ vol.\% MWNTs were slightly decreased by increasing the measuring temperature from 25 to $125^{\circ} \mathrm{C}$ due to the free electrons that dissipate their kinetic energy to atoms as a result of increased collision [20]. Reversely, at high MWNTs content (higher than 1 vol.\% MWNTs), thermal conductivities showed an increase as the measuring temperature increased. Whereas, thermal conductivity of Cu-5 vol.\% MWNTs was relatively increased from 239.1 to $271.7 \mathrm{~W} / \mathrm{mK}$ by increasing the measuring temperature from 25 to $125^{\circ} \mathrm{C}$, respectively. Similar conduct was noted at $\mathrm{Cu} / 10$ vol. MWNTs. This can be attributed to the consistency of high MWNTs thermal conductivity at elevated temperature. However, by increasing MWNTs content more than 1 vol.\%, the measured thermal conductivities of the composites showed deviation compared to the estimated values which were obtained by the rule of mixture (RM), and became even lower than that of the sintered copper. Some researchers have reported similar behavior showing that thermal conductivity of $\mathrm{Cu} / \mathrm{CNTs}$ nanocomposite increased with low CNTs content and then decreased as CNTs content increased [21-24]. For instance, Chu and coworkers [22] have fabricated $\mathrm{Cu} / \mathrm{CNTs}$ nanocomposites using a novel particles compositing method followed by spark plasma sintering. They reported that the addition of CNTs showed no enhancement in the overall thermal conductivity of the fabricated nanocomposites. They suggested that the presence of an interfacial thermal resistance is believed to be the main reason of why the measured thermal conductivities are being degraded $(322 \mathrm{~W} / \mathrm{mK})$. They also attributed these unexpected results to the following factors: low wettability between CNTs and $\mathrm{Cu}$ matrix, nonhomogeneity or bundling of CNTs, porosity and incomplete consolidation processing. Likewise, Kim et al. [23] have reported same underperformance thermal conductivity of Nick-coated SWCNTs/Cu $(150 \mathrm{~W} / \mathrm{mK})$. Yamanaka et al. [24] proposed that the decrease in thermal conductivity is mostly due to the difficulty in dispersing clustered CNTs with an increase in CNTs content. Other similar results were published by Cho and coworker [25]. They used a combination of a wet mixing process accelerated by hetroaggregation method using acid-treated CNTs followed by SPS consolidation technique. They noted that the highest record thermal conductivity was at 1 vol. $\%$ CNTs $(359.2 \mathrm{~W} / \mathrm{mK})$. Their results, however, showed no enhancement in thermal conductivity with CNTs contents higher than 1 vol.\% CNTs. Therefore, our novel technique 
of using functionalized CNTs associate with ultrasonication process followed by MIM method perhaps is the ultimate technology in fabrication of CNTs/MM nanocomposites. This is due to its ability in combining two or more ingredient materials that are not compatible at molten state and difficult to be fabricated via conventional techniques. It also ensures a good dispersion of CNTs in metal matrices by providing high shear forces during mixing process (feedstock preparation).

\section{Conclusions}

For the first time, uniform dispersion of multiwalled carbon nanotube reinforced copper matrix nanocomposites was successfully achieved. The newly developed approach of nanoscale dispersion followed by MIM fabrication technique was proved to be effective in exfoliating the MWNTs clusters and homogeneously distribute the individual MWNT in $\mathrm{Cu}$ matrix. Nanoscale dispersion process provided a functional group on the top surface of MWNTs, which significantly enhanced the solubility of MWNTs in the liquid media of dissolved paraffin wax. These results were verified using EDX, TEM, FESEM, and Raman spectroscope analysis method. EDX and Raman Spectroscope provided sufficient information on the quality of the purification and functionalization process. In addition, it explained the effects of sonication treatment on the MWNTs structure. TEM and FESEM images showed high stability of MWNTs in Cu matrix at high sintering temperature. It also demonstrated a good adherence between dispersed MWNTs and Cu particles. The measured thermal conductivities showed remarkable increase of $11 \%$ with addition of 1 vol.\% MWNTs, and slight decrease at 5 and 10 vol.\% MWNTs compared to the value of pure sintered $\mathrm{Cu}$. Therefore, MIM is considered to be the most promising technique for forming and fabricating high performance heat sink nanocomposites with high precision in shape, high volume production and cost much lower than the traditional methods.

\section{Conflict of Interests}

The authors declare that they have no conflict of interests in this research.

\section{Acknowledgments}

The authors would like to thank the "Universiti Teknologi PETRONAS" for providing funds and laboratory facilities to support this project.

\section{References}

[1] X. Tong Colin, Advanced Materials for Thermal Management of Electronic Packaging, vol. 30 of Advanced Microelectronics, Springer, New York, NY, USA, 2011.

[2] B. Hoefflinger, Chips 2020, Springer, New York, NY, USA, 2012.

[3] L. K. Tan and L. John Johnson, "Metal injection molding of heat sinks," Electronics Cooling Magazine, 2004, http://www .electronics-cooling.com.
[4] J. Kang, J. Li, X. Du, C. Shi, N. Zhao, and P. Nash, "Synthesis of carbon nanotubes and carbon onions by CVD using a $\mathrm{Ni} / \mathrm{Y}$ catalyst supported on copper," Materials Science and Engineering A, vol. 475, no. 1-2, pp. 136-140, 2008.

[5] S. R. B. Arvind Agarwal and D. Lahiri, Carbon Nanotubes Reinforced Metal Matrix Composites. Nanomaterials and Their Applications, CRC Press, New York, NY, USA, 2011, edited by S.E.M. Meyyappan.

[6] S. I. Cha, K. T. Kim, S. N. Arshad, C. B. Mo, and S. H. Hong, "Extraordinary strengthening effect of carbon nanotubes in metal-matrix nanocomposites processed by molecular-level mixing," Advanced Materials, vol. 17, no. 11, pp. 1377-1381, 2005.

[7] K. Chu, H. Guo, C. Jia et al., "Thermal properties of carbon nanotube-copper composites for thermal management applications," Nanoscale Research Letters, vol. 5, no. 5, pp. 868-874, 2010.

[8] G. Chai, Y. Sun, J. Sun, and Q. Chen, "Mechanical properties of carbon nanotube-copper nanocomposites," Journal of Micromechanics and Microengineering, vol. 18, no. 3, Article ID 035013, 2008.

[9] C. Kim, B. Lim, B. Kim et al., "Strengthening of copper matrix composites by nickel-coated single-walled carbon nanotube reinforcements," Synthetic Metals, vol. 159, no. 5-6, pp. 424-429, 2009.

[10] S. Cho, K. Kikuchi, T. Miyazaki, K. Takagi, A. Kawasaki, and T. Tsukada, "Multiwalled carbon nanotubes as a contributing reinforcement phase for the improvement of thermal conductivity in copper matrix composites," Scripta Materialia, vol. 63, no. 4, pp. 375-378, 2010.

[11] K. T. Kim, S. I. Cha, T. Gemming, J. Eckert, and S. H. Hong, "The role of interfacial oxygen atoms in the enhanced mechanical properties of carbon-nanotube-reinforced metal matrix nanocomposites," Small, vol. 4, no. 11, pp. 1936-1940, 2008.

[12] M. Park, B.-H. Kim, S. Kim, D.-S. Han, G. Kim, and K.-R. Lee, "Improved binding between copper and carbon nanotubes in a composite using oxygen-containing functional groups," Carbon, vol. 49, no. 3, pp. 811-818, 2011.

[13] S. Zhang, Y. Shao, G. Yin, and Y. Lin, "Carbon nanotubes decorated with Pt nanoparticles via electrostatic self-assembly: a highly active oxygen reduction electrocatalyst," Journal of Materials Chemistry, vol. 20, no. 14, pp. 2826-2830, 2010.

[14] W. M. Daoush, B. K. Lim, C. B. Mo, D. H. Nam, and S. H. Hong, "Electrical and mechanical properties of carbon nanotube reinforced copper nanocomposites fabricated by electroless deposition process," Materials Science and Engineering A, vol. 513-514, pp. 247-253, 2009.

[15] C. M. Tan, C. Baudot, Y. Han, and H. Jing, "Applications of multi-walled carbon nanotube in electronic packaging," Nanoscale Research Letters, vol. 7, pp. 1-7, 2012.

[16] M.-L. Sham and J.-K. Kim, "Surface functionalities of multiwall carbon nanotubes after UV/Ozone and TETA treatments," Carbon, vol. 44, no. 4, pp. 768-777, 2006.

[17] K. Esumi, M. Ishigami, A. Nakajima, K. Sawada, and H. Honda, "Chemical treatment of carbon nanotubes," Carbon, vol. 34, no. 2, pp. 279-281, 1996.

[18] M. A. Hamon, H. Hui, P. Bhowmik, H. M. E. Itkis, and R. C. Haddon, "Ester-functionalized soluble single-walled carbon nanotubes," Applied Physics A, vol. 74, no. 3, pp. 333-338, 2002.

[19] C.-W. Nan and R. Birringer, "Determining the Kapitza resistance and the thermal conductivity of polycrystals: a simple model," Physical Review B, vol. 57, no. 14, pp. 8264-8268, 1998. 
[20] Y. W. Chung, Introduction to Materials Science and Engineering, CRC Press, 2007.

[21] K. Chu, H. Guo, C. Jia et al., "Thermal properties of carbon nanotube-copper composites for thermal management applications," Nanoscale Research Letters, vol. 5, no. 5, pp. 868-874, 2010.

[22] K. Chu, Q. Wu, C. Jia et al., "Fabrication and effective thermal conductivity of multi-walled carbon nanotubes reinforced $\mathrm{Cu}$ matrix composites for heat sink applications," Composites Science and Technology, vol. 70, no. 2, pp. 298-304, 2010.

[23] C. Kim, B. Lim, B. Kim et al., "Strengthening of copper matrix composites by nickel-coated single-walled carbon nanotube reinforcements," Synthetic Metals, vol. 159, no. 5-6, pp. 424-429, 2009.

[24] S. Yamanaka, R. Gonda, A. Kawasaki et al., "Fabrication and thermal properties of carbon nanotube/nickel composite by spark plasma sintering method," Materials Transactions, vol. 48, no. 9, pp. 2506-2512, 2007.

[25] S. Cho, K. Kikuchi, T. Miyazaki, K. Takagi, A. Kawasaki, and T. Tsukada, "Multiwalled carbon nanotubes as a contributing reinforcement phase for the improvement of thermal conductivity in copper matrix composites," Scripta Materialia, vol. 63, no. 4, pp. 375-378, 2010. 

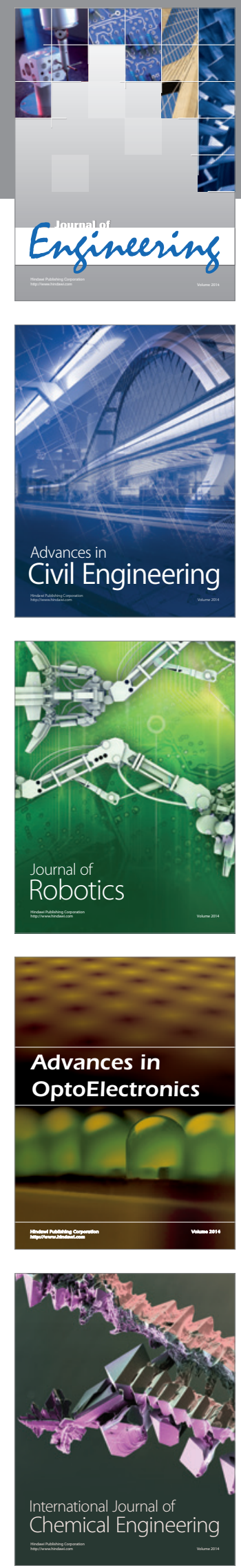

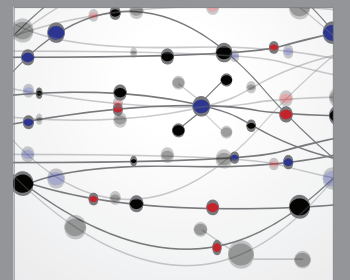

The Scientific World Journal
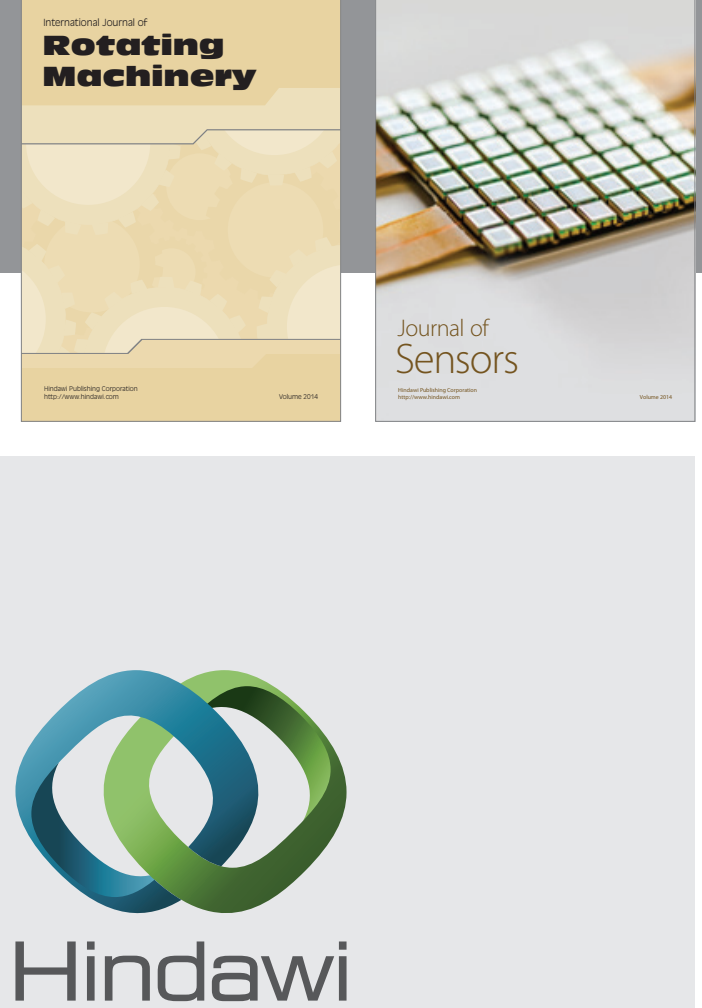

Submit your manuscripts at http://www.hindawi.com
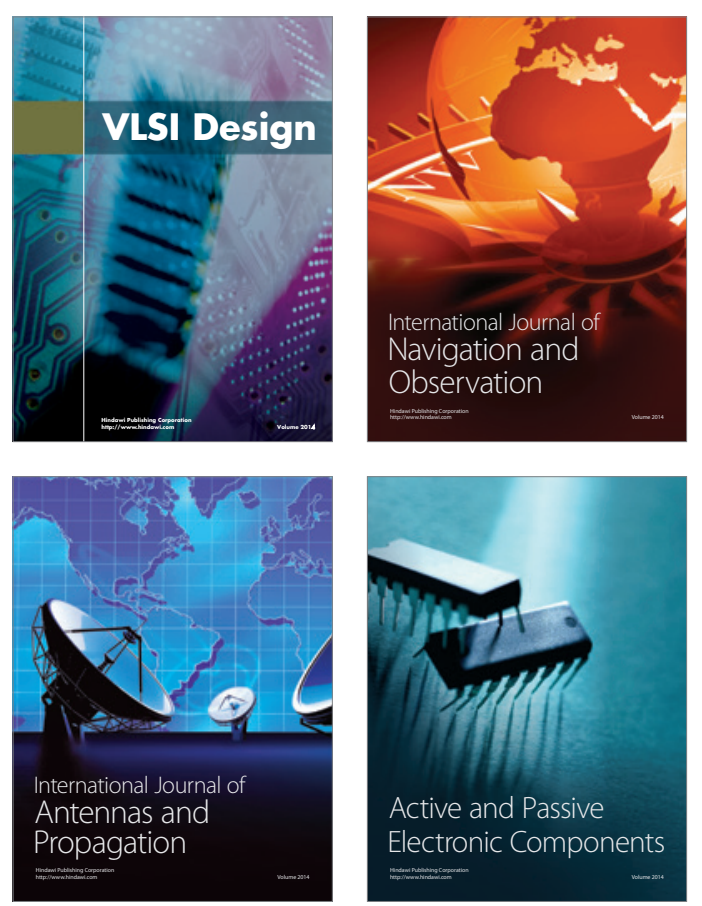
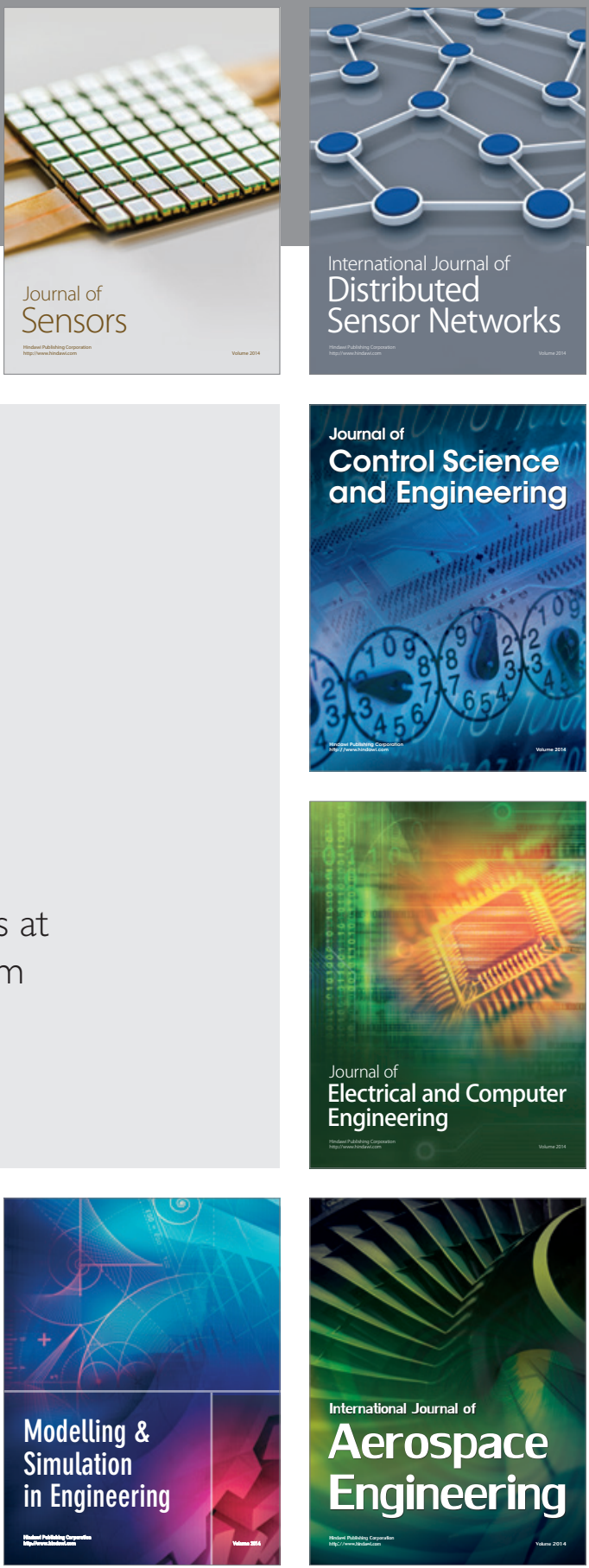

Journal of

Control Science

and Engineering
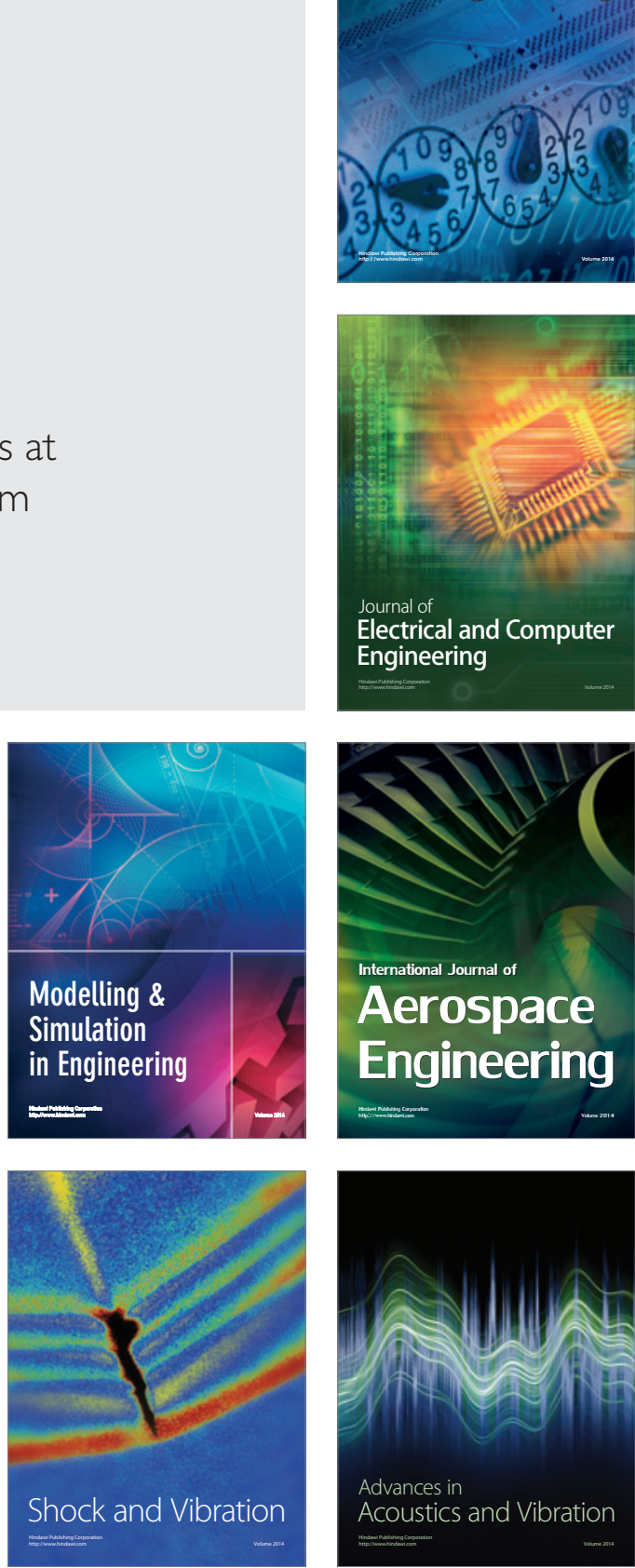